\title{
Power sum decompositions of defining equations of reflection arrangements
}

\author{
Zach Teitler • Alexander Woo
}

Received: 9 May 2013 / Accepted: 3 June 2014 / Published online: 24 June 2014

(C) Springer Science+Business Media New York 2014

\begin{abstract}
We determine the Waring rank and a Waring decomposition of the fundamental skew invariant of any complex reflection group whose highest degree is a regular number. This includes all irreducible real reflection groups.
\end{abstract}

Keywords Waring rank - Reflection group - Skew invariant - Reflection arrangement

Mathematics Subject Classification (2010) Primary 15A21 · 14N15; Secondary 20F55 $\cdot 13 \mathrm{~A} 50 \cdot 15 \mathrm{~A} 69$

Given a homogeneous polynomial $f$ of degree $d$, the Waring rank of $f$, denoted $r(f)$, is the smallest positive integer $r$ such that there exist linear forms $\ell_{1}, \ldots, \ell_{r}$ with $f=\ell_{1}^{d}+\cdots+\ell_{r}^{d}$, and a Waring decomposition is such an expression with length $r=r(f)$. For example, $x y=\frac{1}{4}\left((x+y)^{2}-(x-y)^{2}\right)$, so $r(x y) \leq 2$, and

$$
x y z=\frac{1}{24}\left((x+y+z)^{3}-(x+y-z)^{3}-(x-y+z)^{3}+(x-y-z)^{3}\right),
$$

so $r(x y z) \leq 4$. Waring ranks are notoriously difficult to determine. Even over 160 years after Sylvester's work [32] studying this question, Waring ranks are known only for a few families and particular cases of examples. For a quadratic form, the rank is

\footnotetext{
Z. Teitler $(\bowtie)$

Department of Mathematics, Boise State University, 1910 University Drive, Boise, ID 83725-1555, USA

e-mail: zteitler@boisestate.edu
}

A. Woo

Department of Mathematics, University of Idaho, 875 Perimeter Drive MS 1103, Moscow, ID 83844-1103, USA

e-mail: awoo@uidaho.edu 
equal to the rank of the associated symmetric matrix. Ranks of binary forms have been known since the 19th century; see [10,32]. Ranks of plane cubics such as $x y z$ have been determined by different methods several times, such as by [34] in the 1930s, [9], and [22]. From these results, one can see that in fact $r(x y)=2$ and $r(x y z)=4$.

A theorem of Alexander and Hirschowitz [1] gives the rank of a sufficiently general polynomial of degree $d$ in $n$ variables, meaning one lying in a dense open subset of the space of polynomials. However, it is not known how to determine whether a given form is indeed general, nor is it known in general how to determine the rank of a given form with any reasonable efficiency. Hence there has been a body of recent work determining the rank of certain polynomials of special interest. Some specific ranks were determined in [22], such as $r\left(x\left(y_{1}^{2}+\cdots+y_{m}^{2}\right)\right)=r\left(x\left(y_{1}^{2}+\cdots+y_{m}^{2}+x^{2}\right)\right)=2 m$, $r\left(x^{2} y z\right)=6$, and $r(x y z w)=8$. A result of Ranestad and Schreyer [27] gives a lower bound for rank which turns out to be tight for monomials of the form $\left(x_{1} \cdots x_{n}\right)^{d}$; they have rank $(d+1)^{n-1}$. More generally, the Waring ranks of arbitrary monomials were found by Carlini, Catalisano, and Geramita [7]: $r\left(x_{1}^{a_{1}} \cdots x_{n}^{a_{n}}\right)=\left(a_{2}+1\right) \cdots\left(a_{n}+\right.$ 1) when $a_{1} \leq \cdots \leq a_{n}$. They also found the ranks of sums of pairwise coprime monomials. Among these examples are a few sporadic cases of polynomials having greater rank than that of a general polynomial with the same number of variables and degree; see, for example, [7, Sect. 4.1], [22, Remark 7.3].

One class of polynomials of great interest, generalizing the case of monomials, is the class of products of linear forms, possibly with multiplicities. Geometrically, the vanishing loci of these polynomials are hyperplane (multi)-arrangements. Even in this case, the only products of linear forms for which the Waring rank was previously known are monomials and binary forms.

In this paper, we consider the product of linear forms defining the multi-arrangement associated to a complex reflection group satisfying the technical hypothesis that the highest degree is a regular number. Here, degree means degree of a generator of the invariant ring, as further described in Sect. 1.2, and a regular number is an order of a group element having an eigenvector not lying on any reflection hyperplane; see Sect. 1.3. In particular, our results hold for the reflection arrangement of any irreducible real reflection group. For example, the symmetric group $S_{n}$ acts on $\mathbb{C}^{n}$ as a reflection group with the transposition $t_{i, j}$ acting by reflecting across the hyperplane $H_{i, j}$ defined by $x_{i}-x_{j}=0$. The defining equation of the union of these hyperplanes is $V_{n}=\prod_{i<j}\left(x_{i}-x_{j}\right)$, the classical Vandermonde determinant. This is a form of degree $\left(\begin{array}{l}n \\ 2\end{array}\right)$ in $n$ variables. We show that $r\left(V_{n}\right)=(n-1)$ !.

We briefly sketch our proof here for the case of the Vandermonde determinant. To obtain an expression for $V_{n}$ as a sum of powers of linear forms, one may start

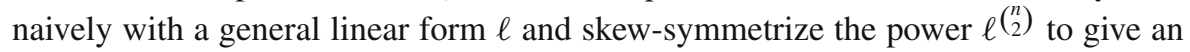
expression of $V_{n}$ as a signed sum of powers involving $\left|S_{n}\right|=n$ ! terms. We improve this by choosing $\ell$ to be a regular eigenvector of a Coxeter element of $S_{n}$, namely a linear form with the $n$-th roots of unity as its coefficients. With this choice, each term in the expression of $V_{n}$ as a sum of $n$ ! powers turns out to be repeated $n$ times. This reduces the number of distinct terms to $(n-1)$ !, so $r\left(V_{n}\right) \leq(n-1)$ !. A lower bound for $r\left(V_{n}\right)$ is given by combining the Ranestad-Schreyer result with well-known facts about the invariant theory of $S_{n}$, yielding $r\left(V_{n}\right) \geq(n-1)$ !. 
The same strategy works for any real or complex reflection group whose largest degree is a regular number. Our main theorem is the following.

Theorem 1 Let $W$ be a finite complex reflection group acting on $\mathbb{C}^{n}$ with degrees $d_{1} \leq \cdots \leq d_{n}$. Suppose that the largest degree $d_{n}$ of $W$ is a regular number. (In particular, this holds for any $W$ that is an irreducible real reflection group.) Let $f$ be the defining equation of the reflection multi-arrangement for $W$. Then $r(f)=$ $d_{1} \cdots d_{n-1}=|W| / d_{n}$.

Additionally we show how to give explicit Waring decompositions in these cases.

We do not assume that $W$ acts essentially on $\mathbb{C}^{n}$.

Other notions of rank are of interest, including cactus rank, smoothable rank, and border rank; see Sect. 1 for details. The lower bound of Ranestad and Schreyer also applies to cactus rank and smoothable rank, enabling us to determine the cactus rank and smoothable rank of the defining equation of the reflection multi-arrangement for any finite complex reflection group. In general, we have the following.

Theorem 2 Let $W$ be a finite complex reflection group with degrees $d_{1} \leq \cdots \leq d_{n}$, and largest regular number $D$. Let $f$ be the defining equation of the reflection multiarrangement for $W$. Then the Waring rank $r(f)$, cactus rank $\operatorname{cr}(f)$, and smoothable $\operatorname{rank} \operatorname{sr}(f)$ satisfy $d_{1} \cdots d_{n-1}=|W| / d_{n}=\operatorname{cr}(f)=\operatorname{sr}(f) \leq r(f) \leq|W| / D$.

In particular, when $d_{n}$ is a regular number, $\operatorname{cr}(f)=\operatorname{sr}(f)=r(f)=|W| / d_{n}$.

We are not, however, able to determine the border ranks of the forms that we consider.

We discuss background both on the aforementioned theorem of Ranestad and Schreyer and on complex reflection groups and their skew invariants in Sect. 1. The proof of our theorem is given in Sect. 2, and Sect. 3 discusses specific examples of reflection groups. It is worth noting that our proof is uniform across types and does not rely on the classification of reflection groups. We work over the complex numbers $\mathbb{C}$.

\section{Background}

\subsection{The Ranestad-Schreyer lower bound}

Now we recall the theorem of Ranestad and Schreyer giving a lower bound for the rank of a homogeneous polynomial $f$ in $n$ variables.

Consider the polynomial ring $S=\mathbb{C}\left[x_{1}, \ldots, x_{n}\right]$. The ring $T=\mathbb{C}\left[\partial_{1}, \ldots, \partial_{n}\right]$ acts on $S$ by differentiation; explicitly, given nonnegative integer vectors $\alpha, \beta \in \mathbb{N}^{n}$,

$$
\partial^{\alpha}\left(\mathbf{x}^{\beta}\right)=\left(\prod_{i=1}^{n} \alpha_{i} !\left(\begin{array}{c}
\beta_{i} \\
\alpha_{i}
\end{array}\right)\right) \mathbf{x}^{\beta-\alpha}
$$

if $\beta-\alpha$ is a nonnegative vector, and $\partial^{\alpha}\left(\mathbf{x}^{\beta}\right)=0$ otherwise, with the action extending $\mathbb{C}$-linearly. This gives rise to the apolar pairing between $S$ and $T$. Letting $S_{k}, T_{k}$ denote the homogeneous pieces of degree $k$, this gives a perfect pairing between $S_{k}$ and $T_{k}$ for each $k$, and a map $S_{d} \otimes T_{a} \rightarrow S_{d-a}$ whenever $d \geq a$, or for all $d$, $a$ with the 
understanding that $S_{k}=T_{k}=0$ when $k<0$. This makes $S$ a $T$-module; it is a graded $T$-module when the grading is reversed (that is, when each $S_{k}$ is given degree $-k$ rather than $k$ ).

Given $f \in S$, define

$$
f^{\perp}=\{D \in T \mid D(f)=0\}
$$

Then $f^{\perp}$ is an ideal of $T$, called the apolar ideal of $f$. The $\operatorname{ring} A^{f}:=T / f^{\perp}$ is a zero-dimensional Gorenstein ring, which is known as the apolar Artinian Gorenstein ring for $f$. If $f$ is a homogeneous polynomial, the ideal $f^{\perp}$ is homogeneous and $A^{f}$ is graded. As a $T$-module, $A^{f}$ is isomorphic to the $T$-submodule of $S$ generated by $f$ (but note that the grading is reversed).

The well-known Apolarity Lemma [17, Lemma 1.31] states that $f$ can be written as a sum of $s$ powers of linear forms if and only if there is an ideal $I \subseteq f^{\perp}$ which is the homogeneous defining ideal of a set of $s$ distinct points in projective space. Thus the Waring rank $r(f)$ is the least $s$ such that $f^{\perp}$ contains the ideal of some set of $s$ reduced points in projective space. Generalizing this notion, the cactus rank (called scheme length in [17]) $\operatorname{cr}(f)$ is the least $s$ such that $f^{\perp}$ contains the saturated ideal of some zero-dimensional scheme of length $s$ in projective space. The smoothable rank $s r(f)$ is the least $s$ such that $f^{\perp}$ contains the saturated ideal of some smoothable zerodimensional scheme of length $s$ in projective space. For more, see [3]. The colorful terminology "cactus rank" was introduced in [27], following [2]. Clearly $\operatorname{cr}(f) \leq$ $s r(f) \leq r(f)$.

A homogeneous ideal $I$ is said to be generated in degree (at most) $\delta$ if there exist polynomials $g_{1}, \ldots, g_{k}$, all of degree at most $\delta$, such that $I=\left\langle g_{1}, \ldots, g_{k}\right\rangle$. The theorem of Ranestad and Schreyer is as follows.

Theorem 3 ([27]) Let $f \in S$ be a homogeneous polynomial, and suppose $f^{\perp}$ is generated in degree $\delta$. Then

$$
c r(f) \geq \frac{\operatorname{dim}_{\mathbb{C}} A^{f}}{\delta} .
$$

The 2006 Ph.D. dissertation of Max Wakefield [33] further investigates the apolar algebras of hyperplane arrangements. In particular, he gives an example of two arrangements with the same combinatorial intersection lattice, only one of which has an apolar ideal that is a complete intersection.

\subsection{Reflection groups and their invariant theory}

We now give a brief overview of the invariant theory of complex reflection groups. Two recent books on this subject are those of Lehrer and Taylor [21] and Kane [19]. A classic reference, addressing only the case of real reflection groups, is that of Humphreys [16]. The statements of this section are originally due to Steinberg [31] and Chevalley [8].

Let $V$ be a $\mathbb{C}$-vector space of dimension $n$. A non-identity element $t \in \mathrm{GL}(V)$ is called a pseudo-reflection if it fixes a hyperplane $H_{t} \in V$ (so it has the eigenvalue 1 
with geometric multiplicity $n-1$ ) and has one eigenvalue which is not equal to 1 . This exceptional eigenvalue must be a $k_{t}$-th root of unity where $k_{t}$ is the order of $t$. A complex reflection group $W$ is a finite subgroup of $\mathrm{GL}(V)$ generated by pseudoreflections. If $V$ can be given a basis such that the matrix representation for every element of $W$ has only real entries, then $W$ is a real reflection group. It is a necessary, but not sufficient, condition for $W$ to be a real reflection group that every pseudoreflection has order 2. The action of $W$ is said to be essential if the only fixed point of $W$ is the origin; equivalently, if the intersection of the reflecting hyperplanes is the origin. We do not assume that $W$ acts essentially.

We now change viewpoints and consider $W$ as an abstract group equipped with a distinguished representation $V$; from this viewpoint $V$ is known as the reflection representation. Note that $V \cong V^{*}$ as representations when $W$ is a real reflection group but not necessarily when $W$ is not.

We identify $\operatorname{Sym}(V)$ with the polynomial ring $S=\mathbb{C}\left[x_{1}, \ldots, x_{n}\right]$ by choosing a basis $x_{1}, \ldots, x_{n}$ of $V$. (Note this is an unusual choice of convention; $S$ is usually identified with $\operatorname{Sym}\left(V^{*}\right)$, but we find it more convenient for our purposes to identify $T$ with $\operatorname{Sym}\left(V^{*}\right)$ and hence $S$ with $\operatorname{Sym}(V)$.) For each reflection $t \in W$, let $L_{t} \in V$ be an exceptional eigenvector of $t$, an eigenvector with eigenvalue $\neq 1$. Now let $f_{W}=\prod L_{t}$, where the product is over all reflections in $W$. (Note that this will usually include more reflections than are necessary to generate $W$.) Let $H_{t} \subset V^{*}$ be the reflecting hyperplane of $t$ in the adjoint action of $W$ on $V^{*}$. Note that $H_{t} \subset V^{*}$ is defined by the linear equation $L_{t}=0$. Two reflections $s, t$ satisfy $H_{s}=H_{t}$ if and only if $L_{s}=L_{t}$ up to a scalar multiple, which occurs if and only if $s$ and $t$ lie in a cyclic subgroup generated by a reflection. Therefore, up to a scalar multiple, $f_{W}=\prod L_{H}^{k_{H}-1}$, where the product is over the hyperplanes $H$ fixed by reflections in $W, L_{H}$ is the complementary exceptional eigenvector of any reflection $t \in W$ fixing $H$, and $k_{H}$ is the order of the cyclic subgroup of reflections fixing $H$. Thus, $f_{W}$ is the defining equation of the reflection multi-arrangement of $W$ in $V^{*}$. (Of course, $f_{W}$ is only defined up to multiplication by a nonzero constant, but this will be irrelevant for our purposes.)

We say that a polynomial $p \in \operatorname{Sym}(V)$ is skew invariant (for $W$ ) if $g \cdot p=$ $(\operatorname{det} g)^{-1} p$ for every $g \in W$. (We take the determinant of $g$ by considering it as an element of GL $(V)$.) The polynomial $f_{W}$ divides every skew invariant polynomial in $S=\operatorname{Sym}(V)$, and we call $f_{W}$ the fundamental skew invariant [19, Prop. 20-1B] [21, Lemma 9.10]. Let $D=\operatorname{deg} f=\sum\left(k_{H}-1\right)$ where the sum is over all reflecting hyperplanes $H$; this is the number of reflections in $W$. As $f_{W}$ divides every skew invariant polynomial, the only skew invariant polynomial of degree less than $D$ is 0 , and every skew invariant polynomial of degree $D$ must be a constant multiple of $f_{W}$. (Note that most sources consider instead elements of $\operatorname{Sym}\left(V^{*}\right)$ on which $W$ acts by det, so they regard $f_{W}$ as the element of $\operatorname{Sym}\left(V^{*}\right)$ defining the reflection multiarrangement in $V$.)

A classical theorem, proved on a case by case basis by Shephard and Todd [30] and uniformly by Chevalley [8], states that the subring $S^{W} \subset S$ of $W$-invariant polynomials is itself a polynomial ring, generated by a set of homogeneous and algebraically independent invariants. These invariants are not uniquely determined, but their degrees 
are. These degrees will be denoted $d_{1}, \ldots, d_{n}$, with $d_{1} \leq \ldots \leq d_{n}$; they are known as the degrees of the complex reflection group $W$. The product of the degrees turns out to be the order of the group $W$; in notation, $\prod_{i=1}^{n} d_{i}=|W|$. The degrees satisfy $\sum_{i=1}^{n}\left(d_{i}-1\right)=D$. Note that 1 is a degree if and only if $W$ does not act essentially.

The fundamental skew invariant has an alternative description as the Jacobian of the invariants; see [19, Prop. 21-1A], [21, Thm. 9.8].

A coinvariant of $W$ is a homogeneous element of $T$ which is invariant under the action of $W$ (induced by the adjoint action on $V^{*}$ ). Let $J_{W}$ be the ideal generated by all positive-degree coinvariants in $T$. Then $J_{W}$ is generated as an ideal by the generators of the coinvariant ring, and these have the same degrees $d_{1}, \ldots, d_{n}$ as the basic invariants in $S$. A theorem of Steinberg [31] states that $J_{W}=f_{W}^{\perp}$ (see also [19, Chapter 26] or [21, Lemma 9.36]) so the apolar ring $A^{f_{W}}$ for the fundamental skew invariant $f_{W}$ is isomorphic to the covariant ring $T / J_{W}$ both as a graded ring and as a representation of $W$. This holds without any assumption that $W$ acts essentially. Since $J_{W}$ is a complete intersection ideal generated in degrees $d_{1}, \ldots, d_{n}, \operatorname{dim}_{\mathbb{C}} T / J_{W}=\prod_{i=1}^{n} d_{i}=|W|$. Thus, we have the following corollary to Theorem 3.

Corollary 4 Let $W$ be a complex reflection group, $f_{W}$ its fundamental skew invariant, and $d_{1} \leq \cdots \leq d_{n}$ its degrees. Then

$$
r\left(f_{W}\right) \geq \operatorname{sr}\left(f_{W}\right)=\operatorname{cr}\left(f_{W}\right)=\frac{d_{1} \cdots d_{n}}{d_{n}}=d_{1} \cdots d_{n-1}=\frac{|W|}{d_{n}} .
$$

Proof The inequalities $r\left(f_{W}\right) \geq s_{r}\left(f_{W}\right) \geq \operatorname{cr}\left(f_{W}\right) \geq|W| / d_{n}$ are immediate from the definitions and Theorem 3. Let $I_{1}, \ldots, I_{n}$ be the invariant generators of $f_{W}^{\perp}=J_{W}$. This is a zero-dimensional complete intersection. Therefore the ideal $\left\langle I_{1}, \ldots, I_{n-1}\right\rangle \subset f_{W}^{\perp}$ is a one-dimensional complete intersection, so it defines a zero-dimensional scheme of length $d_{1} \cdots d_{n-1}=|W| / d_{n}$ in projective space. This shows $\operatorname{cr}\left(f_{W}\right) \leq|W| / d_{n}$. Since every complete intersection is smoothable, $\operatorname{sr}\left(f_{W}\right) \leq|W| / d_{n}$.

In the case where $W$ is the Weyl group for a semisimple Lie group $G$, Borel [6] showed that the covariant ring $A^{f_{W}}$ is the cohomology ring $H^{*}(G / B)$ of the generalized flag variety $G / B$. As a result, $A^{f_{W}}$ has been extensively studied. In particular, $A^{f_{W}}$ comes with a geometrically motivated basis known as the Schubert basis and a set of divided difference operators generating the Schubert basis $[5,11]$. Hiller extended this theory to the case of an arbitrary real reflection group [13,14]. Formulas for the apolar dual basis to the Schubert basis, or, equivalently, the differential operator analogue to the divided difference operators, were given by Postnikov and Stanley [26].

\subsection{Regular numbers}

A vector $v \in V$, where $V$ is the reflection representation, is called a regular vector if $v$ does not lie on any reflecting hyperplane. A group element $g \in W$ is called a regular element if $g$ has a regular eigenvector. A positive integer $d$ is called a regular number if there exists a regular element having a regular eigenvector of eigenvalue a primitive $d$-th root of unity. (Of course if $g$ is a regular element with eigenvalue a primitive $d$-th 
root of unity for a regular eigenvector $v$, then every $d$-th root of unity is an eigenvector for an appropriate power of $g$, on the same eigenvector $v$. Additionally, $g$ has order d.) These definitions are originally due to Springer [29].

The remainder of this section is not necessary for our theorem or its proof, but classifies the groups for which our technical hypothesis requiring that the highest degree be a regular number holds. This classification was also given (with fewer details) by Spaltenstein [28, pp. 305-306].

A reflection group $W$ is irreducible if the reflection representation $V$ is irreducible, or equivalently if $W$ is not the direct product of two proper reflection subgroups.

For an irreducible real reflection group, it was classically known (even before Springer defined the concept) that $d_{n}$ is a regular number. Indeed, pick any chamber (meaning connected component of the complement of the reflection arrangement) $\mathcal{C}$ of $V$ and let $t_{1}, \ldots, t_{n}$ be $n$ reflections such that the linear forms $L_{t_{1}}, \ldots, L_{t_{n}}$ are the hyperplanes bounding $\mathcal{C}$. Then the element $t_{1} t_{2} \cdots t_{n} \in W$ is known as a Coxeter element (and all Coxeter elements are conjugate). The Coxeter element always has order $d_{n}$, and there is a real plane in $V$, usually known as the Coxeter plane, on which it acts by rotation by $2 \pi / d_{n}$; hence it has an eigenvector of eigenvalue $e^{2 \pi i / d_{n}}$ in the complexification of the Coxeter plane. This eigenvector does not lie on a reflecting hyperplane, because the intersection of any reflecting hyperplane with the real Coxeter plane is a real line, so the reflecting hyperplane cannot contain the complex line containing the nonreal eigenvector. See [16, Chap. 3] for details.

More generally, for any irreducible complex reflection group, Lehrer and Springer [20] gave a criterion in terms of degrees and codegrees for a number to be regular. (This result was later given a case-free proof by Lehrer and Michel [12]; see also [21, Sect. 11.4.2].) Consider the action of $W$ on the covariant $\operatorname{ring} \operatorname{Sym}\left(V^{*}\right) / J_{W}$. Let $A_{i}$ be the degree $i$ graded piece of $\operatorname{Sym}\left(V^{*}\right) / J_{W}$. Given any representation $U$ of $W$, we define the fake degree of $U$ to be the generating function

$$
g_{U}(t)=\sum_{i \geq 0}\left(\operatorname{dim}_{\mathbb{C}} \operatorname{Hom}_{\mathbb{C W}}\left(A_{i}, U\right)\right) t^{i}
$$

We can write $g_{U}(t)$ in the form

$$
g_{U}(t)=t^{e_{1}(U)}+\cdots+t^{e_{r}(U)},
$$

with $e_{1}(U) \leq e_{2}(U) \leq \cdots \leq e_{r}(U)$. The numbers $e_{1}(U), \ldots, e_{r}(U)$ are known as the $U$-exponents. In the case where $U=V$ is the reflection representation, these are known simply as the exponents, and it is classically known that $e_{i}=d_{i}-1$ and hence that $\sum_{i=1}^{n} e_{n}$ is the degree of $f_{W}$. In the case $U=V^{*}$, the $V^{*}$-exponents are known as the coexponents, and the numbers $d_{i}^{*}=e_{i}\left(V^{*}\right)-1$ are the codegrees.

Now the criterion of Lehrer and Springer is as follows.

Theorem 5 ([20]) A number $d$ is regular if and only if the number of degrees that $d$ divides is equal to the number of codegrees $d$ divides, or, in notation,

$$
\#\left\{i: d \mid d_{i}\right\}=\#\left\{i: d \mid d_{i}^{*}\right\} .
$$


Suppose $W$ acts essentially, so $J_{W}$ contains no elements of degree 1 . Hence $A_{1}$, the degree 1 piece of $\operatorname{Sym}\left(V^{*}\right) / J_{W}$, is $V^{*}$. Therefore $e_{1}\left(V^{*}\right)=1$ and $d_{1}^{*}=0$. Hence, every regular number divides at least one codegree, so every regular number divides at least one degree, and in particular no regular number exceeds the largest degree $d_{n}$. If $W$ acts inessentially on $V$, with fixed subspace $V_{0}$, the largest degree of the action on $V$ is equal to the largest degree of the essential action on $V / V_{0}$, and the actions on $V$ and on $V / V_{0}$ have the same regular numbers, so for the inessential action on $V$ once again no regular number exceeds the largest degree.

Using this criterion, one can easily calculate using the Shephard-Todd classification and a table of degrees and codegrees (for example in [21, Appendix D.2]) that the only irreducible reflection groups whose largest degrees are not regular numbers are the sporadic group $G_{15}$ and the imprimitive groups $G(d e, e, n)$ where $d, e, n \geq 2$ and at least one of $e>2$ or $n>2$.

For a reducible reflection group $W=W_{1} \times W_{2}$, it follows easily from the definitions that a number is regular for $W$ if and only if it is regular for each of $W_{1}$ and $W_{2}$, while the degrees of $W$ are simply the union (as a multiset) of the degrees of $W_{1}$ and of $W_{2}$. These facts imply that a reducible reflection group has its highest degree regular if and only if the highest degrees of its irreducible components are equal, and this common highest degree is regular for each of the components. Note also that $f_{W_{1} \times W_{2}}=f_{W_{1}} f_{W_{2}}$.

\section{Proof of main theorem}

Now we give upper bounds for $r\left(f_{W}\right)$ by giving expressions for $f_{W}$ as sums of powers of linear forms.

For a polynomial $p \in S=\operatorname{Sym}(V)$, the skew-symmetrization of $p$ is

$$
\operatorname{alt}(p):=\frac{1}{|W|} \sum_{w \in W}(\operatorname{det} w)(w \cdot p) .
$$

Note that for $w \in W, w \cdot \operatorname{alt}(p)=(\operatorname{det} w)^{-1} \operatorname{alt}(p)$, so alt $(p)$ is skew invariant for any $p$.

Recall that if $p \in S$ is a skew invariant polynomial, then $f_{W}$ divides $p$. In particular, if $D=\sum_{i=1}^{n}\left(d_{i}-1\right)$ is the degree of $f_{W}$, and $p$ has degree $D$, then alt $(p)$ must be a (possibly zero) scalar multiple of $f_{W}$. Therefore, given any linear form $L \in V$, we see that $\operatorname{alt}\left(L^{D}\right)$ is a (possibly zero) scalar multiple of $f_{W}$.

We now show the following lemma.

Lemma 6 With $D$ as above, the polynomial $\operatorname{alt}\left(L^{D}\right)$ is zero if and only if L lies on a reflecting hyperplane in $V$.

Proof Let $\mathbb{C}_{\operatorname{det}^{-1}}$ be the rank one representation of $W$ on which $w \in W$ acts as $(\operatorname{det} w)^{-1}$, where the determinant is given by the determinant of $w$ in its action on the reflection representation $V$. Then the skew-symmetrization operator alt is a $\mathbb{C} W$ linear map alt $: \operatorname{Sym}^{D}(V) \rightarrow \mathbb{C}_{\mathrm{det}^{-1}}$, where $\mathbb{C}_{\mathrm{det}^{-1}}$ is identified with the span of $f_{W}$. We can tensor this map by the determinant representation $\mathbb{C}_{\text {det }}$ to get a map alt $\otimes \mathbb{C}_{\text {det }}: \operatorname{Sym}^{D}(V) \otimes \mathbb{C}_{\text {det }} \rightarrow \mathbb{C}$, which we can identify with an invariant element 
of $\operatorname{Sym}^{D}\left(V^{*}\right) \otimes \mathbb{C}_{\operatorname{det}^{-1}}$, or equivalently an element of $\operatorname{Sym}^{D}\left(V^{*}\right)$ on which $W$ acts by det.

Just as $f_{W}$ divides every element of $\operatorname{Sym}(V)$ on which $W$ acts by $\operatorname{det}^{-1}$, we have a corresponding element $f_{W}^{*} \in \operatorname{Sym}\left(V^{*}\right)$ (defined only up to a nonzero scalar multiple), vanishing precisely on the reflecting hyperplanes $H_{t}^{*} \subset V$, each to order $k_{t}-1$, that divides every element of $\operatorname{Sym}\left(V^{*}\right)$ on which $W$ acts by det.

The operator alt is clearly nonzero, $\operatorname{since} \operatorname{alt}(p)=p$ for the polynomials $p$ on which $w \cdot p=(\operatorname{det} w)^{-1} p$ for all $w \in W$, in particular for $p=f_{W}$. Hence, regarded as a tensor, alt is a nonzero multiple of $f_{W}^{*}$, so alt $\left(L^{D}\right)$ is zero if and only if the tensor $f_{W}^{*}$ vanishes on $L^{D}$, if and only if the polynomial $f_{W}^{*}$ vanishes on $L$, if and only if $L$ lies on a reflecting hyperplane.

For the case of a real reflection group with no $H_{4}$ factors, a stronger result is known. A linear form $L$ in a graded Artinian Gorenstein ring $A$ with socle in degree $D$ is a strong Lefschetz element if multiplication by $L^{D-2 i}$ induces an isomorphism between the graded pieces $A_{i}$ and $A_{D-i}$ for all $i$ with $0 \leq i \leq\left\lfloor\frac{D}{2}\right\rfloor$. Since $\mathbb{C}_{\mathrm{det}^{-1}}$ can be identified with the socle of the coinvariant ring (thought of as a quotient of $S$ rather than $T$, which is not a problem since $V \cong V^{*}$ in the case of a real reflection group) so that alt is the natural projection from $S_{D} \rightarrow\left(S / J_{W}\right)_{D}$, a strong Lefschetz element $L$ for the coinvariant ring automatically satisfies alt $\left(L^{D}\right) \neq 0$. Maeno, Numata, and Wachi [25] show that $L$ is a strong Lefschetz element for the coinvariant ring of a real reflection group if and only if $L$ does not lie on a reflecting hyperplane (except for groups with $\mathrm{H}_{4}$ factors, which they leave as a conjecture). For Weyl groups, they use the Hard Lefschetz theorem describing strong Lefschetz elements in cohomology rings of algebraic varieties; for the remaining cases they make explicit calculations. It should also be possible to derive similar results from the work of McDaniel [24].

Now suppose $L$ is an eigenvector of $w \in W$ with eigenvalue $e^{\frac{2 \pi i}{d}}$. Let $C_{w} \subset W$ be the subgroup generated by $w$, and let $W / C_{w}$ denote the collection of its left cosets. Note $\left|C_{w}\right|$ is some multiple of $d$ and hence $\left|W / C_{w}\right| \leq|W| / d$. Picking any representative $\sigma$ for each coset in $W / C_{w}$, we have

$$
\begin{aligned}
\operatorname{alt}\left(L^{D}\right) & =\frac{1}{|W|} \sum_{\sigma \in W / C_{w}}(\operatorname{det} \sigma)\left(\sigma \cdot \sum_{v \in C_{w}}(\operatorname{det} v)\left(v \cdot L^{D}\right)\right) \\
& =\frac{1}{|W|} \sum_{\sigma \in W / C_{w}}(\operatorname{det} \sigma)\left(\sigma \cdot \sum_{j=0}^{\left|C_{w}\right|-1}(\operatorname{det} w)^{j} e^{2 \pi i D j / d} L^{D}\right) \\
& =\frac{1}{|W|}\left(\sum_{j=0}^{\left|C_{w}\right|-1}(\operatorname{det} w)^{j} e^{2 \pi i D j / d}\right) \sum_{\sigma \in W / C_{w}}(\operatorname{det} \sigma)\left(\sigma \cdot L^{D}\right)
\end{aligned}
$$

which is a sum of at most $\left|W / C_{w}\right| \leq|W| / d$ powers of linear forms. Hence we have shown the following proposition. 
Proposition 7 Let $W$ be a complex reflection group, $f_{W}$ its fundamental skew invariant, and $d$ a regular number for $W$. Then

$$
r\left(f_{W}\right) \leq \frac{|W|}{d} .
$$

Combining Proposition 7 and Corollary 4, we have shown our main theorem:

Theorem 8 Let $W$ be a finite complex reflection group acting on $\mathbb{C}^{n}, d_{1} \leq \cdots \leq d_{n}$ the degrees of $W$, and suppose that $d_{n}$ is a regular number. Then $\operatorname{cr}\left(f_{W}\right)=\operatorname{sr}\left(f_{W}\right)=$ $r\left(f_{W}\right)=|W| / d_{n}$.

See [3] for additional related quantities that are between the Waring rank and cactus rank, and therefore equal in the case of this theorem.

Consider the factor $\sum_{j=0}^{\left|C_{w}\right|-1}(\operatorname{det} w)^{j} e^{2 \pi i D j / d}$ appearing in (2). Observe that det $w$ is a $\left|C_{w}\right|$-th root of unity, as is $e^{2 \pi i D / d}$, since $d$ divides $\left|C_{w}\right|$. Let $\zeta=(\operatorname{det} w) e^{2 \pi i D / d}$. We are considering the factor $\sum_{j=0}^{\left|C_{w}\right|-1} \zeta^{j}$, which is $\left|C_{w}\right|$ if $\zeta=1$, otherwise 0 . When $L$ is a regular vector, $\operatorname{alt}\left(L^{D}\right) \neq 0$, so necessarily $\zeta=1$ and the sum is $\left|C_{w}\right|$. Then the explicit expression simplifies to

$$
\operatorname{alt}\left(L^{D}\right)=\frac{\left|C_{w}\right|}{|W|} \sum_{\sigma \in W / C_{w}}(\operatorname{det} \sigma)(\sigma \cdot L)^{D} .
$$

Hence

$$
f_{W}=C \sum_{\sigma \in W / C_{w}}(\operatorname{det} \sigma)(\sigma \cdot L)^{D},
$$

for some nonzero scalar $C$. Note that $f_{W}$ is only defined up to a scalar factor.

\section{Examples}

\subsection{The Vandermonde determinant}

The classical Vandermonde determinant $\prod_{1<i<j<n}\left(x_{i}-x_{j}\right)$ is the fundamental skew invariant of the action of $W=S_{n}$ on $\mathbb{C}^{n}$ by permuting coordinates. This is an irreducible real reflection group with reflection hyperplanes defined by the polynomials $x_{i}-x_{j}$ for $i \neq j$; hence its fundamental skew invariant is the Vandermonde determinant $f_{S_{n}}=\prod_{i<j}\left(x_{i}-x_{j}\right)$, of degree $\left(\begin{array}{l}n \\ 2\end{array}\right)$.

This action is non-essential as it fixes the line spanned by $(1, \ldots, 1)$. Passing to an essential action, say by restricting to the subspace defined by $x_{1}+\cdots+x_{n}=0$, would correspond to changing $f_{S_{n}}$ by a substitution such as replacing $x_{n}$ with $-x_{1}-\cdots-x_{n-1}$.

The degrees (other than 1) are $2,3, \ldots, n$, and since $S_{n}$ is an irreducible real reflection group, the highest degree $n$ is a regular number. Explicitly, the linear form $L=\sum_{j=1}^{n} e^{2 \pi i(j-1) / n} x_{j}$ is a regular element for which the $n$-cycle $(12 \cdots n)$ acts by multiplication by $e^{2 \pi i / n}$. 
This implies that the Waring rank of the Vandermonde determinant is $(n-1)$ !, with an explicit expression given by

$$
\begin{aligned}
\prod_{i<j}\left(x_{i}-x_{j}\right) & =C \sum_{\sigma \in S_{n} / C_{n}} \operatorname{sgn}(\sigma)(\sigma \cdot L)^{\left(\begin{array}{c}
n \\
2
\end{array}\right)} \\
& =C \sum_{\sigma \in S_{n} / C_{n}} \operatorname{sgn}(\sigma)\left(\sum_{j=1}^{n} e^{2 \pi i(j-1) / n} x_{\sigma(j)}\right)^{\left(\begin{array}{c}
n \\
2
\end{array}\right)},
\end{aligned}
$$

for some scalar $C$, see below, where $C_{n}$ is the cyclic subgroup generated by $(12 \cdots n)$ and $\sigma$ denotes a choice of coset representatives in $S_{n} / C_{n}$. For example, the sum can be taken over permutations $\sigma$ such that $\sigma(1)=1$.

The cactus rank and smoothable rank are also equal to $(n-1)$ !.

By the Alexander-Hirschowitz theorem, the rank of a general form of degree $\left(\begin{array}{l}n \\ 2\end{array}\right)$ in $n$ variables is

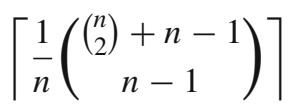

which is greater than $(n-1)$ ! for $n>1$. Thus, the Vandermonde determinant $f_{S_{n}}$ has less than general rank, and in fact the ratio of $r\left(f_{S_{n}}\right)$ to the general rank rapidly approaches zero as $n$ goes to infinity.

If we work instead with the essential action of $S_{n}$ on $\mathbb{C}^{n-1}$, the rank of the fundamental skew invariant should be compared to the generic rank of a form in one less variable. This still grows far more rapidly than $r\left(f_{S_{n}}\right)$.

Now we find the scalar $C$ in (4). Consider the monomial $x_{1}^{0} x_{2}^{1} \cdots x_{n}^{n-1}$. Its coeffi-

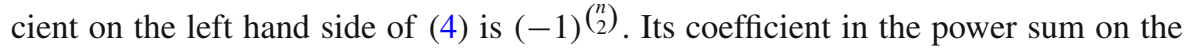
right hand side is

$$
\sum_{\sigma \in S_{n} / C_{n}}(\operatorname{sgn} \sigma)\left(\begin{array}{c}
\left(\begin{array}{l}
n \\
2
\end{array}\right) \\
1,2, \ldots, n-1
\end{array}\right) \prod_{j=1}^{n}\left(e^{2 \pi i\left(\sigma^{-1}(j)-1\right) / n}\right)^{j-1}
$$

We fix the choice of coset representatives to be the set of $\sigma \in S_{n}$ such that $\sigma(1)=1$. For convenience let $\alpha$ be a primitive $n$-th root of unity, say $e^{2 \pi i / n}$. Let $M_{n}$ be the

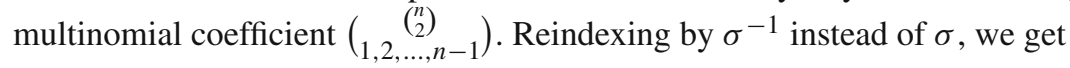

$$
\begin{aligned}
\frac{1}{C}=(-1)^{\left(\begin{array}{l}
n \\
2
\end{array}\right)} M_{n} \sum_{\substack{\sigma \in S_{n} \\
\sigma(1)=1}}(\operatorname{sgn} \sigma) \prod_{j=2}^{n} \alpha^{(j-1)(\sigma(j)-1)} \\
=(-1)^{\left(\begin{array}{c}
n \\
2
\end{array}\right)} M_{n} \operatorname{det}\left(\begin{array}{cccc}
\alpha & \alpha^{2} & \ldots & \alpha^{n-1} \\
\alpha^{2} & \alpha^{4} & \ldots & \alpha^{2(n-1)} \\
\vdots & \vdots & & \vdots \\
\alpha^{n-1} & \alpha^{2(n-1)} & \ldots & \alpha^{(n-1)^{2}}
\end{array}\right)
\end{aligned}
$$




$$
\begin{aligned}
& =(-1)^{\left(\begin{array}{l}
n \\
2
\end{array}\right)} M_{n} \alpha^{\left(\begin{array}{l}
n \\
2
\end{array}\right)} \prod_{1 \leq j<k \leq n-1}\left(\alpha^{k}-\alpha^{j}\right) \\
& =(-1)^{\left(\begin{array}{c}
n \\
2
\end{array}\right)+n+1} M_{n} \prod_{1 \leq j<k \leq n-1}\left(\alpha^{k}-\alpha^{j}\right) .
\end{aligned}
$$

We now need to calculate $\prod_{1 \leq j<k \leq n-1}\left(\alpha^{k}-\alpha^{j}\right)$, which we denote by $P$. We will calculate $|P|$ and $\arg P$ separately. This calculation has most likely been known since the nineteenth century, especially the calculation of $P^{2}$, which is (up to sign) the discriminant of the polynomial $x^{n-1}+x^{n-2}+\cdots+x+1$, but we could not find a simple reference. Let $V$ be the Vandermonde matrix

$$
V=\left(\begin{array}{ccccc}
1 & 1 & 1 & \ldots & 1 \\
\alpha & \alpha^{2} & \alpha^{3} & \ldots & \alpha^{n-1} \\
\alpha^{2} & \alpha^{4} & \alpha^{6} & \ldots & \alpha^{2(n-1)} \\
\vdots & \vdots & \vdots & & \vdots \\
\alpha^{n-2} & \alpha^{2(n-2)} & \alpha^{3(n-2)} & \ldots & \alpha^{(n-1)(n-2)}
\end{array}\right)
$$

so that $P=\operatorname{det} V$. Then $|P|^{2}=\operatorname{det}\left(V V^{\dagger}\right)$, where $V^{\dagger}$ is the conjugate transpose of $V$. It is easy to see that

$$
V V^{\dagger}=\left(\begin{array}{ccccc}
n-1 & -1 & -1 \ldots & -1 \\
-1 & n-1 & -1 \ldots & -1 \\
\vdots & \vdots & \vdots & \vdots \\
-1 & -1 & -1 \ldots & n-1
\end{array}\right)
$$

The determinant of this matrix may be computed easily in several ways, for example, by noticing that it is a particularly simple circulant matrix. One amusing way is to note that this is the deleted Laplacian of the complete graph on $n$ vertices, so by the MatrixTree Theorem, $\operatorname{det}\left(V V^{\dagger}\right)$ counts the number of spanning trees of the complete graph. Spanning trees of the complete graph are equivalent to trees on $n$ labeled vertices, and there are $n^{n-2}$ such trees. Hence $|P|=\sqrt{n^{n-2}}$.

Since $P^{2}>0, \arg P$ must be a multiple of $\pi / 2$. To calculate $\arg P$, we sum up $\arg \left(\alpha^{k}-\alpha^{j}\right)$ for $1 \leq j<k \leq n-1$. An easy computation shows

$$
\alpha^{k}-\alpha^{j}=e^{\frac{i \pi(k+j)}{n}}\left(2 i \sin \frac{(k-j) \pi}{n}\right) .
$$

Hence

$$
\arg \left(\alpha^{k}-\alpha^{j}\right)=\frac{\pi(j+k)}{n}+\frac{\pi}{2}
$$


and

$$
\begin{aligned}
\arg P & =\sum_{1 \leq j<k \leq n-1}\left(\frac{\pi(j+k)}{n}+\frac{\pi}{2}\right) \\
& =\pi\left(\sum_{1 \leq j<k \leq n-1} \frac{j+k}{n}\right)+\frac{(n-1)(n-2) \pi}{4} .
\end{aligned}
$$

Each value $\ell \in\{1, \ldots, n-1\}$ appears as $k$ in $\ell-1$ terms of this sum and as $j$ in $n-1-\ell$ terms, for a total of $n-2$ appearances.

$$
\begin{aligned}
\arg P & =\pi\left(\sum_{\ell=1}^{n-1} \frac{(n-2) \ell}{n}\right)+\frac{(n-1)(n-2) \pi}{4} \\
& =\frac{3(n-1)(n-2) \pi}{4} .
\end{aligned}
$$

Hence, $P=(-i)\left(\begin{array}{c}n-1 \\ 2\end{array}\right) \sqrt{n^{n-2}}$, and

$$
\frac{1}{C}=i^{\left(\begin{array}{c}
n-1 \\
2
\end{array}\right)} M_{n} \sqrt{n^{n-2}} .
$$

Therefore, we have the following expression for the Vandermonde determinant.

$$
\prod_{i<j}\left(x_{i}-x_{j}\right)=\frac{(-i)^{\left(\begin{array}{c}
n-1 \\
2
\end{array}\right)}}{\left(\begin{array}{c}
\left(\begin{array}{c}
n \\
2
\end{array}\right) \\
1,2, \ldots, n-1
\end{array}\right) \sqrt{n^{n-2}}} \sum_{\sigma \in S_{n} / C_{n}} \operatorname{sgn}(\sigma)\left(\sum_{j=1}^{n} e^{2 \pi i(j-1) / n} x_{\sigma(j)}\right)^{\left(\begin{array}{c}
n \\
2
\end{array}\right)}
$$

We note two related alternative methods for determining the nonzero scalar $C$. First, one can use the divided difference operator $\partial_{w_{0}}$ associated to the greatest element (in Bruhat order) $w_{0}$ of the group $W$. Explicitly, for any homogeneous form $p$ of degree $\left(\begin{array}{l}n \\ 2\end{array}\right)$, we have

$$
|W| \operatorname{alt}(p)=\partial_{w}(p) \prod_{1 \leq i<j \leq n}\left(x_{i}-x_{j}\right)
$$

See [23] for details; the above equation is Proposition 2.3.2. Alternatively, one can use the differential operator equivalent to this divided difference operator. This operator is an explicitly known (at least for Weyl groups) multiple of the fundamental skew invariant in $T$; see [18, Thm. 1.1] or [26, Cor. 9.2]. We see no reason to believe either method would produce a simpler calculation. 


\subsection{Types B and D}

The group $B_{n}$ of order $2^{n} n$ ! acts on $\mathbb{C}^{n}$, and its fundamental skew invariant is $f_{B_{n}}=$ $\prod_{i=1}^{n} x_{i} \prod_{i<j}\left(x_{i}-x_{j}\right)\left(x_{i}+x_{j}\right)$, of degree $n+2\left(\begin{array}{l}n \\ 2\end{array}\right)=n^{2}$. Its highest degree is $2 n$. In the realization of $B_{n}$ as signed permutations, one choice of regular element of order $2 n$ is the signed permutation $v \in B_{n}$ with $v(i)=i+1$ for $1 \leq i<n$ and $v(n)=\overline{1}$, so in its action on $\mathbb{C}^{n}, v \cdot x_{i}=x_{i+1}$ for $i<n$ and $v \cdot x_{n}=-x_{1}$. The linear form $L=\sum_{j=1}^{n} e^{2 \pi i(j-1) / 2 n} x_{j}$ is a regular form on which $v$ acts by multiplication by $e^{2 \pi i / 2 n}$.

Thus the rank $r\left(f_{B_{n}}\right)=2^{n-1}(n-1)$ !, and an explicit expression is given by

$$
\prod_{i=1}^{n} x_{i} \prod_{i<j}\left(x_{i}-x_{j}\right)\left(x_{i}+x_{j}\right)=C \sum_{\sigma \in B_{n} / C_{n}} \operatorname{sgn}(\sigma)(\sigma \cdot L)^{n^{2}},
$$

for some nonzero scalar $C$, where $C_{n}$ is the cyclic subgroup generated by $v$ and the $\sigma$ are coset representatives for $B_{n} / C_{n}$. For example we may take $\left\{\sigma \in B_{n} \mid \sigma(1)=1\right\}$.

The group $D_{n}$ of order $2^{n-1} n$ ! acts on $\mathbb{C}^{n}$ as a real reflection group with fundamental skew invariant $f_{D_{n}}=\prod_{i<j}\left(x_{i}-x_{j}\right)\left(x_{i}+x_{j}\right)$, of degree $2\left(\begin{array}{l}n \\ 2\end{array}\right)=n(n-1)$. Its highest degree (assuming $n \geq 3$ ) is $2 n-2$. Thus $r\left(f_{D_{n}}\right)=2^{n-2} n(n-2)$ !. One choice of regular element of order $2 n-2$ is the signed permutation $v \in D_{n}$ with $v(1)=\overline{1}, v(i)=i+1$ for $2 \leq i \leq n-1$, and $v(n)=\overline{2}$, so in its action on $\mathbb{C}^{n}, v \cdot x_{1}=-x_{1}, v \cdot x_{i}=x_{i+1}$ when $2 \leq i \leq n-1$, and $v \cdot x_{n}=-x_{2}$. The linear form $L=\sum_{j=2}^{n} e^{2 \pi i(j-2) /(2 n-2)} x_{j}$ is a regular form for which $v$ acts by multiplication by $e^{2 \pi i /(2 n-2)}$. Thus

$$
\prod_{i<j}\left(x_{i}-x_{j}\right)\left(x_{i}+x_{j}\right)=C \sum_{\sigma \in D_{n} / C_{n}} \operatorname{sgn}(\sigma)(\sigma \cdot L)^{n(n-1)},
$$

for some nonzero scalar $C$, where $C_{n}$ is the cyclic subgroup generated by $v$ and the $\sigma$ are coset representatives for $D_{n} / C_{n}$.

As these groups are irreducible real reflection groups, the cactus, smoothable, and Waring ranks are equal.

By the Alexander-Hirschowitz theorem, the rank of a general form of degree $n^{2}$, respectively $n(n-1)$, in $n$ variables is

$$
\left\lceil\frac{1}{n}\left(\begin{array}{c}
n^{2}+n-1 \\
n-1
\end{array}\right)\right\rceil, \quad \text { respectively } \quad\left\lceil\frac{1}{n}\left(\begin{array}{c}
n(n-1)+n-1 \\
n-1
\end{array}\right)\right\rceil,
$$

and these are greater than $2^{n-1}(n-1)$ !, respectively $2^{n-2} n(n-2)$ !. Again $f_{B_{n}}$ and $f_{D_{n}}$ have less than general rank, and ratios of their ranks to the general ranks go to zero. 


\subsection{Exceptional groups}

We list the fundamental skew invariant, its degree, and its rank for the exceptional real reflection groups $E_{8}, E_{7}, E_{6}, F_{4}, H_{4}$, and $H_{3}$. Here, we regard $E_{7}$ and $E_{6}$ as acting non-essentially on $\mathbb{C}^{8}$ (as subgroups of $E_{8}$ ).

The fundamental skew invariants of these groups are in Table 1 , with $\beta=\cos (\pi / 5)$.

In Table 2, we list, for each group, the number of variables, degree, and rank of its fundamental skew invariant, along with the general rank for forms in that number of variables and of that degree and the quotient of the rank by the general rank. Since we have written $f_{E_{7}}$ and $f_{E_{6}}$ as polynomials in 8 variables, we have compared their ranks with the general ranks of forms in that many variables. One may instead pass to 7 or 6 variables, respectively.

Table 1 Fundamental skew invariants for exceptional groups

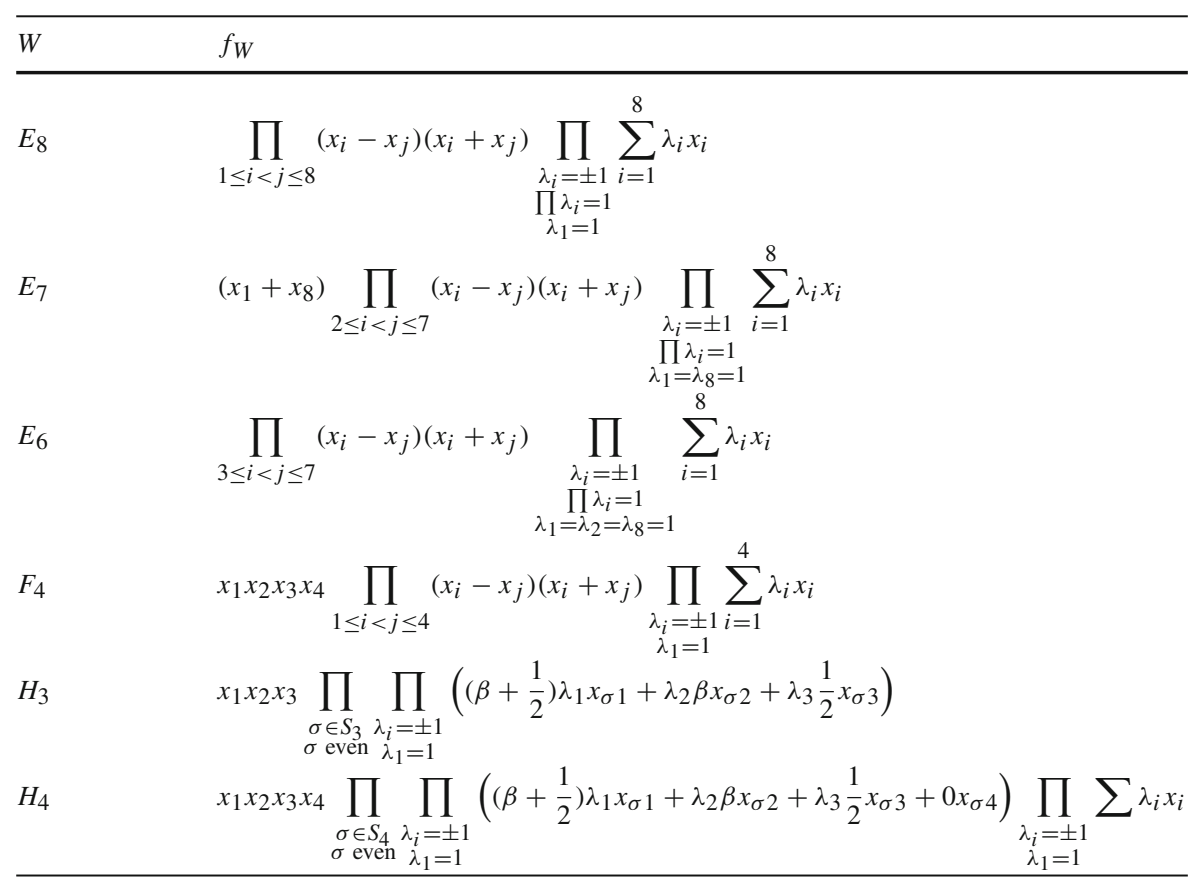

Table 2 Comparison of ranks for skew invariants of real exceptional groups with general rank

\begin{tabular}{llllll}
\hline$W$ & Variables & $\operatorname{deg}\left(f_{W}\right)$ & $r\left(f_{W}\right)$ & General rank & Quotient \\
\hline$E_{8}$ & 8 & 120 & $64 \times 9 !$ & 11169551972 & $2.08 \times 10^{-3}$ \\
$E_{7}$ & 8 & 63 & $4 \times 8 !$ & 149846840 & $1.08 \times 10^{-3}$ \\
$E_{6}$ & 8 & 36 & $6 \times 6 !$ & 4028015 & $1.07 \times 10^{-3}$ \\
$F_{4}$ & 4 & 24 & 96 & 732 & 0.131 \\
$H_{4}$ & 4 & 60 & 480 & 9928 & 0.0242 \\
$H_{3}$ & 3 & 15 & 12 & 46 & 0.261 \\
\hline
\end{tabular}


Finally, briefly, the dihedral groups $I_{2}(m)$ (including $G_{2}=I_{2}(6)$ ) have fundamental skew invariant $f_{I_{2}(m)}=x^{m}-y^{m}$ in a suitable choice of coordinates, whence the Waring rank is clearly 2 .

\subsection{Imprimitive groups}

We find the following rank of a natural generalization of the Vandermonde determinant, and of the case $D_{n}$ :

$$
r\left(\prod_{1 \leq i<j \leq n}\left(x_{i}^{e}-x_{j}^{e}\right)\right)=e^{n-2} n(n-2) ! .
$$

Similarly, generalizing the case $B_{n}$, we find:

$$
r\left(\prod_{i=1}^{n} x_{i}^{d-1} \prod_{1 \leq i<j \leq n}\left(x_{i}^{d}-x_{j}^{d}\right)\right)=d^{n-1}(n-1) ! .
$$

Indeed, these polynomials are the fundamental skew invariants of certain imprimitive reflection groups. See [21, Chapter 2] for information on these groups. These groups are denoted $G(d e, e, n)$ for positive integers $d, e, n$ with $n \geq 2$. By [21, Lemma 2.8], the fundamental skew invariant of $G(d e, e, n)$ is

$$
\prod_{i} x_{i}^{d-1} \prod_{i<j}\left(x_{i}^{d e}-x_{j}^{d e}\right) .
$$

This has degree $n(d-1)+\left(\begin{array}{l}n \\ 2\end{array}\right) d e$. The polynomials displayed above are the fundamental skew invariants of $G(e, e, n)$ and $G(d, 1, n)$. (Note that $A_{n}=G(1,1, n), B_{n}=$ $G(2,1, n)$, and $D_{n}=G(2,2, n)$.)

The degrees of $G(d e, e, n)$ are $d e, 2 d e, \ldots,(n-1) d e$, and $n d$. The largest degree is a regular number if and only if $d=1, e=1$, or $e=n=2$ : that is, for the groups $G(e, e, n), G(d, 1, n)$, and $G(2 d, 2,2)$. We exclude the cases $n \leq 2$ since the resulting polynomials are binary and their ranks are known by Sylvester's results. If $e=1$ then the largest degree is $n d$ and it is a regular number. Hence the Waring rank (and cactus rank and smoothable rank) of the fundamental skew invariant is

$$
(d)(2 d) \cdots((n-1) d)=d^{n-1}(n-1) ! .
$$

If $e>1$ then $d=1$, the largest degree is $(n-1) e$, and it is a regular number. Hence the Waring rank (and cactus rank and smoothable rank) of the fundamental skew invariant is

$$
(e)(2 e) \cdots((n-2) e)(n)=e^{n-2} n(n-2) ! \text {. }
$$




\subsection{Monomials}

The monomial $x_{1}^{a_{1}} \cdots x_{n}^{a_{n}}, a_{1} \leq \cdots \leq a_{n}$, is the fundamental skew invariant for the reflection group $\mathbb{Z} /\left(a_{1}+1\right) \mathbb{Z} \times \cdots \times \mathbb{Z} /\left(a_{n}+1\right) \mathbb{Z}$, where the generator of the $j$-th component $\mathbb{Z} /\left(a_{j}+1\right) \mathbb{Z}$ acts on $x_{j}$ by multiplication by $e^{-2 \pi i /\left(a_{j}+1\right)}$.

The degrees for this group are $d_{i}=a_{i}+1$. We immediately recover the result of Ranestad and Schreyer that the rank, smoothable rank, and cactus rank satisfy

$$
r\left(x_{1}^{a_{1}} \cdots x_{n}^{a_{n}}\right) \geq \operatorname{sr}\left(x_{1}^{a_{1}} \cdots x_{n}^{a_{n}}\right)=\operatorname{cr}\left(x_{1}^{a_{1}} \cdots x_{n}^{a_{n}}\right)=\left(a_{1}+1\right) \cdots\left(a_{n-1}+1\right),
$$

see [27]. However, all of the codegrees are 0 ; since the representation $V^{*}$ decomposes into $n$ distinct 1-dimensional representations, we have

$$
\operatorname{dim}_{\mathbb{C}} \operatorname{Hom}\left(V^{*}, \operatorname{Sym}^{1}\left(V^{*}\right)\right)=\operatorname{dim}_{\mathbb{C}} \operatorname{Hom}\left(V^{*}, V^{*}\right)=n
$$

so all the coexponents are 1 . Therefore, according to Theorem 5, the only case where the largest degree $a_{n}+1$ is a regular number is when all the $a_{i}$, and hence all the degrees, are equal.

Alternatively, note that the regular numbers of $\mathbb{Z} /\left(a_{i}+1\right) \mathbb{Z}$ are just the divisors of $a_{i}+1$, and recall that the regular numbers of a reducible reflection group are precisely the numbers that are regular for each of the irreducible components. Thus, the largest regular number of $\mathbb{Z} /\left(a_{1}+1\right) \mathbb{Z} \times \cdots \times \mathbb{Z} /\left(a_{n}+1\right) \mathbb{Z}$ is $\operatorname{gcd}\left(a_{1}+1, \ldots, a_{n}+1\right)$. Again, this is equal to the largest degree $a_{n}+1$ if and only if all the $a_{i}$ are equal.

In particular, we recover the observation of Ranestad and Schreyer that $r\left(\left(x_{1} \cdots x_{n}\right)^{d}\right)=(d+1)^{n-1}[27]$.

For other monomials, the lower bound of Ranestad and Schreyer is not tight, and in most cases, our upper bound also is not tight. Indeed, as mentioned in the introduction, Carlini, Catalisano, and Geramita show that $r\left(x_{1}^{a_{1}} \cdots x_{n}^{a_{n}}\right)=\left(a_{2}+1\right) \cdots\left(a_{n}+1\right)$ when $a_{1} \leq \cdots \leq a_{n}$ [7]. Regarding upper bounds, our construction in Proposition 7 gives a tight upper bound only in the case $a_{1}+1$ divides $a_{j}+1$ for all $j$.

See [7, Sect. 4.1] and [15] for a comparison of the ranks of monomials with general ranks.

It is interesting to note that the explicit expression for $x_{1}^{a_{1}} \cdots x_{n}^{a_{n}}$ as a sum of powers given in [4] (which is not the only such expression) is exactly the skew-symmetrization of $\left(x_{1}+\cdots+x_{n}\right)^{D}, D=a_{1}+\cdots+a_{n}$, over the subgroup $\{1\} \times \mathbb{Z} /\left(a_{2}+1\right) \mathbb{Z} \times$ $\cdots \times \mathbb{Z} /\left(a_{n}+1\right) \mathbb{Z}$ rather than over the whole group. Furthermore, $x_{1}+\cdots+x_{n}$ is not necessarily an eigenvector of any element in the group, depending on whether the $a_{i}+1$ have a common divisor.

The proof of Theorem 5 by Lehrer and Michel [12] and the determination of the ranks of monomials in $[4,7]$ both involve the algebraic geometry of the covariant ring, so we speculate that combining these ideas appropriately could lead to a method to determine the rank of the fundamental skew invariant for an arbitrary complex reflection group even in the case where the largest degree is not a regular number. 
Acknowledgments We thank Vic Reiner and Hirotachi Abo for helpful comments. We also thank the anonymous referees for numerous helpful comments and suggestions which significantly improved the paper. AW is partially supported by NSA Young Investigators Grant H98230-13-1-0242.

\section{References}

1. Alexander, J., Hirschowitz, A.: Polynomial interpolation in several variables. J. Algebr. Geom. 4(2), 201-222 (1995). MR 1311347 (96f:14065)

2. Buczyńska, W., Buczyński, J.: Secant varieties to high degree veronese reembeddings, catalecticant matrices and smoothable Gorenstein schemes. J. Algebr. Geom. 23, 63-90 (2014)

3. Bernardi, A., Brachat, J., Mourrain, B.: A comparison of different notions of ranks of symmetric tensors, http://arxiv.org/abs/1210.8169v2 [math.AG], (2012)

4. Buczyńska, W., Buczyński, J., Teitler, Z.: Waring decompositions of monomials. J. Algebra 378, 45-57 (2013). MR 3017012

5. Bernšteřn, I.N., Gel'fand, I.M., Gel'fand, S.I.: Schubert cells, and the cohomology of the spaces $G / P$. Uspehi Mat. Nauk 28 3(171), 3-26 (1973). MR 0429933 (55 \#2941)

6. Borel, A.: Sur la cohomologie des espaces fibrés principaux et des espaces homogènes de groupes de Lie compacts. Ann. Math. 2(57), 115-207 (1953). MR 0051508 (14,490e)

7. Carlini, E., Catalisano, M.V., Geramita, A.V.: The solution to the Waring problem for monomials and the sum of coprime monomials. J. Algebra 370, 5-14 (2012)

8. Chevalley, Claude: Invariants of finite groups generated by reflections. Am. J. Math. 77, 778-782 (1955). MR 0072877 (17,345d)

9. Comon, P., Mourrain, B.: Decomposition of quantics in sums of powers of linear forms, Signal Processing, Elsevier 53(2), (1996)

10. Comas, G., Seiguer, M.: On the rank of a binary form. Found. Comput. Math. 11(1), 65-78 (2011). MR 2754189

11. Demazure, Michel.: Désingularisation des variétés de Schubert généralisées, Ann. Sci. École Norm. Sup. 4(7): 53-88, Collection of articles dedicated to Henri Cartan on the occasion of his 70th birthday, I. (1974)

12. Gustav, I.: Lehrer and Jean Michel, Invariant theory and eigenspaces for unitary reflection groups. C. R. Math. Acad. Sci. Paris 336(10), 795-800 (2003). MR 1990017 (2004d:13005)

13. Hiller, H.L.: Schubert calculus of a Coxeter group. Enseign. Math. 27(1-2), 57-84 (1981). MR 6309620 (82m:14031)

14. Hiller, H.: Geometry of Coxeter groups, Research Notes in Mathematics, vol. 54. Pitman (Advanced Publishing Program), Boston (1982). MR 649068 (83h:14045)

15. Holmes, E., Plummer, P., Siegert, J., Teitler, Z.: Maximum Waring ranks of monomials, http://arxiv. org/abs/1309.7834 [math.AG], (2014)

16. Humphreys, J.E.: Reflection Groups and Coxeter Groups, Cambridge Studies in Advanced Mathematics. Cambridge University Press, Cambridge (1990)

17. Iarrobino, A., Kanev, V.: Power Sums, Gorenstein Algebras, and Determinantal Loci, Lecture Notes in Mathematics, vol. 1721. Springer, Berlin (1999). Appendix C by Iarrobino and Steven L. Kleiman. MR 1735271 (2001d:14056)

18. John, R.: Stembridge, A weighted enumeration of maximal chains in the Bruhat order. J. Algebr. Combin. 15(3), 291-301 (2002). MR 1900629 (2003e:05149)

19. Kane, R.: Reflection Groups and Invariant Theory, CMS Books in Mathematics/Ouvrages de Mathématiques de la SMC, 5. Springer, New York (2001). MR 1838580 (2002c:20061)

20. Lehrer, G.I., Springer, T.A.: Reflection subquotients of unitary reflection groups. Canad. J. Math. 51(6), 1175-1193 (1999). Dedicated to H. S. M. Coxeter on the occasion of his 90th birthday. MR 1756877 (2007f:20082)

21. Lehrer, G.I., Taylor, D.E.: Unitary Reflection Groups, Australian Mathematical Society Lecture Series, vol. 20. Cambridge University Press, Cambridge (2009). MR 2542964 (2010j:20056)

22. Landsberg, J.M., Teitler, Zach: On the ranks and border ranks of symmetric tensors, Found. Comp. Math. 10, 3, 339-366 (2010)

23. Manivel, L.: Symmetric functions, Schubert polynomials and degeneracy loci, SMF/AMS Texts and Monographs, vol. 6, American Mathematical Society, Providence, RI; Société Mathématique de France, 
Paris, Translated from the 1998 French original by John R. Swallow, Cours Spécialisés [Specialized Courses], 3. (2001)

24. McDaniel, C.: The strong Lefschetz property for coinvariant rings of finite reflection groups. J. Algebra 331, 68-95 (2011). MR 2774648 (2012b:13021)

25. Maeno, T., Numata, Y., Wachi, A.: Strong Lefschetz elements of the coinvariant rings of finite Coxeter groups. Algebr. Represent. Theory 14(4), 625-638 (2011). MR 2817446 (2012e:20086)

26. Postnikov, A., Stanley, R.P.: Chains in the Bruhat order. J. Algebraic Combin. 29(2), 133-174 (2009). MR 2475632 (2011a:14100)

27. Ranestad, K., Schreyer, F.O.: On the rank of a symmetric form. J. Algebra 346, 340-342 (2011). MR 2842085

28. Spaltenstein, N.: Coxeter classes of unitary reflection groups. Invent. Math. 119(2), 297-316 (1995). MR 1312502 (96g:20059)

29. Springer, T.A.: Regular elements of finite reflection groups. Invent. Math. 25, 159-198 (1974). MR 0354894 (50 \#7371)

30. Shephard, G.C., Todd, J.A.: Finite unitary reflection groups. Can. J. Math. 6, 274-304 (1954). MR $0059914(15,600 \mathrm{~b})$

31. Steinberg, R.: Differential equations invariant under finite reflection groups. Trans. Am. Math. Soc. 112, 392-400 (1964). MR 0167535 (29 \#4807)

32. Sylvester, J.J.: An essay on canonical forms, supplement to a sketch of a memoir on elimination, transformation and canonical forms, originally published by George Bell, Fleet Street, London, 1851. Paper 34 in Mathematical Papers, Vol. 1. Chelsea, New York (1973). Originally published by Cambridge University Press in 1904., 1851

33. Wakefield, M.: On the derivation module and apolar algebra of an arrangement of hyperplanes, Ph.D. thesis. University of Oregon, Eugene (2006)

34. Yerushalmy, J.: On the configuration of the nine base points of a pencil of equianharmonic cubics. Am. J. Math. 54(2), 279-284 (1932). MR MR1506892 